Open Access

\title{
Perceived risk of watery diarrhea and dysentery and intended compliance with chemoprophylaxis among a deployed military population
}

\author{
Chad K. Porter ${ }^{1 *}$, Kristen Felicione ${ }^{2}$, David R. Tribble ${ }^{3}$, Adam W. Armstrong $^{4}$, Manal Mostafa ${ }^{5}$ and Mark S. Riddle ${ }^{1}$
}

\begin{abstract}
Background: Infectious diseases are a leading cause of morbidity among travelers to resource-limited regions and primary prevention is a cornerstone to risk reduction. Chemoprophylaxis has been successfully utilized for specific diseases.

Methods: We assessed self-reported compliance to daily chemoprophylaxis among deployed US military personnel. A 21 item self-completed questionnaire was completed by military personnel during mid-deployment.

Results: The perception of high disease risk was associated with an increased likelihood of compliance with daily chemoprophylaxis. However, 60 \% of respondents stated they would not comply with a daily regimen.

Conclusions: These data highlight the complexity of perceived risk and the difficulties with prophylactic interventions.
\end{abstract}

\section{Background}

Infectious diseases have been and remain a leading cause of morbidity in travelers, including the deployed military. In military populations, disease prevention has emphasized preparation, education, personal protective measures, vaccines, and chemoprophylaxis [1]. The effectiveness of these measures is often dependent on the disease in question, though understanding the perceptions and attitudes towards primary prevention strategies may enable the development of more targeted interventions with higher success rates.

Vaccination has a clear history of reducing disease risk for many diseases of military importance; however, for several (malaria, travelers' diarrhea, dengue fever, skin infections, etc.) vaccines remain unavailable. In their absence, increased use of personal protection, vector control, and chemoprophylaxis has reduced malaria incidence in endemic regions [2]. In contrast, chemoprophylaxis is not currently recommended for travelers' diarrhea (TD), and utilization of standard public health practices to minimize disease risks has yielded little impact on TD incidence,

\footnotetext{
* Correspondence: chad.k.porter2.civ@mail.mil

${ }^{1}$ Enteric Diseases Department, Naval Medical Research Center, Silver Spring, MD, USA

Full list of author information is available at the end of the article
}

with average monthly attack rates of $30 \%$ among deployed populations over the past two decades [3, 4]. In addition to the burden of acute illness, evidence is increasing on the importance of several sequelae of TD [5]. This, coupled with the development of new non-absorbable antibiotics, may modify the paradigm for chemoprophylaxis [6]. In fact, such a study was recently conducted in active duty military personnel deployed to Incirlik Air Base [7]. While the data from that study highlight the potential efficacy of a specific chemoprophylactic regimen in certain regions, it is unclear whether comparable effectiveness results would be borne out due to potential issues with non-compliance. As such, we sought to assess factors associated with intended compliance to a daily regimen of chemoprophylaxis for TD as well as malaria (for comparison).

\section{Methods}

This was a cross-sectional study utilizing a convenience sample of active duty US military personnel deployed to Iraq, Afghanistan, and the surrounding region during an in-processing brief as part of rest and recuperation (R\&R) at Camp As Sayliyah in Doha, Qatar as previously described [4]. From April 2006 through September 2007, a single page, 21 item, self-completed questionnaire 
soliciting information on demographics, prior deployments, perceived disease risk and likelihood of compliance to a daily prophylactic was distributed to military personnel. The questionnaires were voluntary and anonymous and were designed to assess potential future compliance with a hypothetical travelers' diarrhea prophylactic compared to theoretical compliance with malaria prophylaxis. Data were entered into Microsoft Excel (Microsoft Inc., Redmond, WA) and verified by Naval Medical Research Unit No. 3 (NAMRU-3) (Cairo, Egypt) staff.

Descriptive analyses were performed using Student's $t$ test for continuous variables and Pearson Chi-Square tests for categorical variables. Perceived disease risk was assessed by the question "What is your perceived risk of acquiring Disease X". Logistic regression models were developed using backwards elimination to identify factors associated with an increased likelihood of compliance to daily chemoprophylaxis for TD as well as malaria. All variables with a $\mathrm{p}<0.25$ were retained in the final model. Statistical significance was set at an alpha $=$ 0.05 and all analyses were conducted using SAS v9.3 (Cary, NC).

\section{Human subjects protection}

This study was approved by the institutional review board at the NAMRU-3, Cairo, Egypt.

\section{Results}

A total of 2235 questionnaires were distributed, completed and returned. Subjects were predominantly male (84.2 \%), in the Army (76.3 \%) and enlisted (87.3\%). The mean age of responders was 28.9 years. The majority of respondents $(71.8 \%)$ were deployed in support of Operation Iraqi Freedom and were comprised of active duty (62.9 \%), reserve (18.5\%) and National Guard (17.1\%) personnel. These demographic and populations characteristics were representative of the deployment population to the region.
A significant proportion of subjects identified themselves as being at no risk for dysentery (46.7), watery diarrhea (45.8), or malaria (48.6) regardless of the country to which the subject was predominately deployed, while approximately $30 \%$ did not know their risk for these infectious diseases (Table 1). Perceived disease risk varied by country of deployment with the highest rates of 'high risk' observed in individuals deployed to Afghanistan. The majority of subjects reported they would not take a daily chemoprophylactic to prevent diarrhea (61.5\%) or malaria (62.2\%) though subjects were most likely (always or very likely) to comply with daily chemoprophylaxis against malaria (17.1\%) rather than for diarrhea (10.9 \%) (data not shown).

After controlling for covariates, a high perceived risk of diarrhea and malaria was associated with a significant increase in the likelihood of compliance with daily chemoprophylaxis compliance (OR: 3.8 and 4.9, respectively) (Table 2). Additionally, military officers were more likely to comply with daily chemoprophylaxis for diarrhea and malaria compared to enlisted respondents. Similarly, those in the Navy and Air Force were more likely to comply with chemoprophylaxis against malaria compared to those in the Army.

\section{Discussion}

The majority of respondents indicated no perceived risk to the diseases of interest including dysentery or watery diarrhea. Recent studies have estimated travelers' diarrhea rates among deployed military personnel to be approximately 30 cases per 100 person-months [3]. Because of the consistent and fairly high prevalence of TD among similar populations, the low proportion perceiving watery diarrhea and dysentery as "high risk" (less than $5 \%$ ) is discordant with anticipated responses, and it may be possible the term "risk" was perceived to mean something other than the potential for incident illness. This is supported by the minimal difference in perceived high risk of watery diarrhea and malaria; a disease with a

Table 1 Reported perceived disease risk among responders

\begin{tabular}{|c|c|c|c|c|c|}
\hline \multirow[t]{2}{*}{ Country predominately deployed } & \multirow[t]{2}{*}{ Disease } & \multicolumn{4}{|c|}{ Perceived Risk, n(\%) } \\
\hline & & High risk & Low risk & No risk & Don't know/missing \\
\hline \multirow[t]{3}{*}{ Afghanistan } & Malaria & $28(9.7)$ & $100(34.7)$ & $96(33.3)$ & $64(22.2)$ \\
\hline & Dysentery & $19(6.6)$ & $91(31.6)$ & $101(35.1)$ & $77(26.7)$ \\
\hline & Watery diarrhea & $26(9.0)$ & $81(28.1)$ & $100(34.7)$ & $81(28.1)$ \\
\hline \multirow[t]{3}{*}{ Iraq } & Malaria & $11(0.7)$ & $294(18.6)$ & $801(50.7)$ & $473(30.0)$ \\
\hline & Dysentery & $35(2.2)$ & $300(19.0)$ & $773(49.0)$ & $471(29.8)$ \\
\hline & Watery diarrhea & $51(3.2)$ & $292(18.5)$ & $759(48.5)$ & $477(30.2)$ \\
\hline \multirow[t]{3}{*}{ Other/Unknown } & Malaria & $4(1.1)$ & $78(21.2)$ & $189(51.4)$ & $97(26.4)$ \\
\hline & Dysentery & $5(1.4)$ & $84(22.8)$ & $170(46.2)$ & 109 (29.6) \\
\hline & Watery diarrhea & $12(3.3)$ & 91 (24.7) & $164(44.6)$ & $101(27.5)$ \\
\hline
\end{tabular}


Table 2 Multivariate analyses of factors associated with the likelihood of compliance to a once-a-day pill for prevention of diarrhea or malaria

\begin{tabular}{lll}
\hline Significant Risk Factors & \multicolumn{2}{l}{ OR $(95 \%$ Cl) of increased likelihood of } \\
& \multicolumn{1}{c}{ compliance with daily chemoprophylaxis } \\
\cline { 2 - 3 } & Diarrhea & Malaria \\
\hline Perceived High Risk & $3.78(2.44-5.92)$ & $4.88(2.52-9.46)$ \\
Officer rank & $1.61(1.09-2.37)$ & $2.21(1.59-3.06)$ \\
Branch & - & \\
Army & & $1.00($ ref $)$ \\
Air Force & $2.79(1.55-5.03)$ \\
Marines & & $0.64(0.40-1.03)$ \\
Navy & & $1.62(1.11-2.37)$ \\
\hline
\end{tabular}

Hosmer and Lemeshow Goodness-of-fit chi-square of 0.31 and 1.43 for diarrhea and malaria models, respectively

much lower incidence rate; though it is notable that malaria risks in the two theaters of operation is different [4].

In addition to low rates of perceived high disease risk, a high proportion of respondents reported they would never take a daily pill to prevent diarrhea or malaria. For malaria, these estimates are consistent with historical reports of low chemoprophylaxis compliance; however, targeted intervention efforts have been shown to increase compliance rates and this is why directly observed therapy has been put into practice in the Department of Defense [8]. Chemoprophylaxis is not currently recommended for TD, though the development of new non-absorbable antibiotics and risk of post-infectious sequelae may modify the paradigm [9]. Nonetheless, given the challenges associated with behavioral modification, a major emphasis on prevention should continue to be vaccination when feasible.

It is unclear if increased perceived disease risk would meaningfully modify the likelihood of taking daily chemoprophylaxis. For example, a population of vacationers traveling for a significantly shorter period of time compared to active duty military members may perceive greater disease risk, thus resulting in increased compliance of daily chemoprophylaxis. Additionally, it is unclear if increased knowledge regarding the relationships between illness prevention, infection prevention, and theoretical sequelae prevention would alter attitudes toward daily chemoprophylaxis. Furthermore, the reasons for variability in compliance across military-specific characteristics are unclear highlight an area for future study. If prevention of TD is shown in future studies to decrease sequalae, issues of long-term daily chemoprophylaxis compliance could be explored in deployment settings.

\section{Conclusions}

Given the overall low perceived risk of diarrhea and the strong association between a high perceived disease risk and compliance, daily chemoprophylaxis may be a suboptimal means of disease prevention. Additional studies are needed to better explore the knowledge, attitudes and practice of US military personnel as well as other travel populations.

\section{Competing interest \\ The authors declare that they have no competing interests.}

\section{Authors' contributions}

MR, AA, DT designed and carried out the study. MM was involved in data entry and management. CKP and KF carried out all analyses. All authors were involved in interpreting the results of the study. All authors read and approved the final manuscript.

\section{Acknowledgements}

\section{Disclaimer}

The views expressed in this article are those of the authors and do not necessarily reflect the official policy or position of the Department of the Navy, Department of Defense, nor the U.S. Government. This is a US Government work. There are no restrictions on its use. There were no financial conflicts of interests among any of the authors. This study was conducted under support of the Military Infectious Disease Research Program and Department of Defense Global Emerging Infections Surveillance and Response System funding.

\section{Copyright statement}

Some authors are employees of the U.S. Government and military service members. This work was prepared as part of official duties. Title 17 U.S.C. $\S 105$ provides that "Copyright protection under this title is not available for any work of the United States Government." Title 17 U.S.C. \$101 defines a U.S. Government work as a work prepared by a military service member or employee of the U.S. Government as part of that person's official duties.

\section{Author details}

${ }^{1}$ Enteric Diseases Department, Naval Medical Research Center, Silver Spring, MD, USA. ${ }^{2}$ George Washington University, School of Public Health and Health Services, Washington, District of Colombia, USA. ${ }^{3}$ Infectious Diseases Clinical Research Program, Uniformed Services University, Bethesda, MD, USA. ${ }^{4}$ United States Naval Medical Research Unit No.6, Lima, Peru. ${ }^{5}$ United States Naval Medical Research Unit No. 3, Cairo, Egypt.

Received: 3 March 2015 Accepted: 29 July 2015

Published online: 19 August 2015

\section{References}

1. Murray CK, Horvath LL. An approach to prevention of infectious diseases during military deployments. Clin Infect Dis. 2007;44(3):424-30.

2. Armed Forces Health Surveillance. C., Update: malaria, U.S. Armed Forces, 2012. MSMR. 2013;20(1):2-5. discussion 5.

3. Riddle MS et al. Incidence, etiology, and impact of diarrhea among longterm travelers (U.S. military and similar populations): A systematic review. Am J Trop Med Hyg. 2006;74(5):891-900.

4. Sanders JW et al. The epidemiology of self-reported diarrhea in operations Iraqi freedom and enduring freedom. Diagn Microbiol Infect Dis. 2004;50(2):89-93.

5. Porter CK, Thura N, Riddle MS. Quantifying the incidence and burden of postinfectious enteric sequelae. Mil Med. 2013;178(4):452-69.

6. Alajbegovic $S$ et al. Effectiveness of rifaximin and fluoroquinolones in preventing travelers' diarrhea (TD): a systematic review and meta-analysis. Syst Rev. 2012;1(1):39.

7. Armstrong AW et al. A randomized, double-blind, placebo-controlled study evaluating the efficacy and safety of rifaximin for the prevention of travelers' diarrhea in US military personnel deployed to Incirlik Air Base, Incirlik, Turkey. J Travel Med. 2010;17(6):392-4.

8. Brisson $\mathrm{P}$, Woll M, Brisson M. Improving compliance with malaria chemoprophylaxis in Afghanistan. Mil Med. 2012;177(12):1539-42.

9. DuPont $\mathrm{HL}$ et al. Expert review of the evidence base for prevention of travelers' diarrhea. J Travel Med. 2009;16(3):149-60. 